\title{
Confocal Raman-in-SEM Imaging: a New Method for 3D Morphology of Asbestos- like Fibers in a Mineral Matrix - Complementarity with SEM-FIB.
}

\author{
Guillaume Wille $^{1}$, Didier Lahondere ${ }^{1}$, Ute Schmidt ${ }^{2}$, Jeromine Duron ${ }^{1}$, Rostislav Vana ${ }^{3}$, Jeremie \\ Silvent $^{4}$ and Xavier Bourrat ${ }^{1}$ \\ 1. BRGM, French Geological Survey, Orleans, France. \\ 2. WITec Gmbh, Ulm, Germany. \\ 3. TESCAN, Brno, Czech Republic \\ ${ }^{4}$ TESCAN ORSAY HOLDING France, Fuveau, France
}

Asbestos consists of natural mineral fibers crystallized in a specific way with specific properties including flexibility, high tensile strength, resistance to heat and chemical degradation. The term asbestos refers to one fibrous serpentine, and five asbestiform varieties of amphiboles [1][2]. Asbestos is considered a Category 1 human carcinogen (e.g. [1][3][4][5][6]), as inhalation of asbestos fibers causes respiratory diseases, in particular asbestosis, lung cancers and malignant mesothelioma. Due to the pathogenicity of asbestos fibers, their use has been banned in most countries around the world. The classification as "asbestos" comprises three main characteristics: sub-micrometric fibrous morphology (so-called asbestiform), chemistry and crystallography (i.e. mineralogy).

The ban applies to natural materials, which are quarried and used for public works projects. Compliance with existing rules requires accurate diagnosis of the asbestos or non-asbestos nature of the materials likely to be exploited in a quarry or involved in the opening of a construction site. Conventional methods for asbestos diagnosis, mainly based on the use of TEM, are hard to apply to massive materials as the sample preparation protocol is complex, it is likely to release artifacts like cleavage fragments, or sample-piece thinning is costly and hard to achieve and thus not applicable in routine analysis.

The use of a LV-FE-SEM-EDS (Low vacuum field emission gun scannning electron microscope equiped with energy dispersive spectroscopy) coupled to confocal Raman-in-SEM imaging (RISE) is a new and efficient method for identifying the mineral nature of sub-micrometer fibers or fibrous bundles. SEM coupled to EDS provides a qualitative or semi-quantitative composition of the fiber. The crystal structure is determined by confocal Raman spectroscopy (with lateral resolution down to $360 \mathrm{~nm}$ [7]). Thus the combination of these two kinds of information allows for accurate identification of both the nature and the morphology of the mineral. The combination of SEM, EDS and confocal Raman imaging in a unique analytical system (RISE) allows precise location of the same area / fiber and identification of its mineral nature. In addition, RISE allows the acquisition of 3D data, which is able to provide morphological information of the mineral distribution in the sample volume and leading to the determination of the aspect ratio, a critical parameter for asbestos (i.e. asbestiform or non-asbestiform). Moreover, this combination of techniques is not destructive. The coupling of SEM-EDS with RISE is a powerful analytical system that simplifies and reinforces existing analytical procedures.

For highest resolution studies of fibers in the sample volume (3 D imaging), the RISE can be connected to a FIB-SEM [7]. Although FIB volume reconstruction is a destructive method, the resolution of the voxel can be extended to below $100 \mathrm{~nm}$ [8] - a resolution sufficient for visualizing even the thinnest fibers. Furthermore, Raman 3D view is an efficient tool for sampling TEM slices using the FIB. 


\section{References}

[1] WHO (World Health Organization) Asbestos and other natural mineral fibres, Environmental Health criteria 53, Geneva (1986)

[2] NIOSH (National Institute for Occupational Safety and Health) Asbestos fibers and other elongate mineral particles: State of roadmap for research. Current Intelligence Bulletin 62, (2011).

[3] IARC (International Agency for Research on Cancer) IARC Monographs on the evaluation of carcinogenic risks of chemicals to humans. Silica and some silicates, 42, Lyon, France. (1987)

[4] IARC (International Agency for Research on Cancer) IARC Monographs on the evaluation of carcinogenic risks to humans. Arsenic, Metals, Fibres and Dusts, 100C, Lyon, France. (2012)

[5] HSE (Health and Safety Executive) Asbestos: The analysts' guide for sampling, analysis and clearance procedures (2006).

[6] Kazan-Allen L Asbestos and mesothelioma: Worldwide trends. Lung Cancer, 49 Suppl.1 (2005), p. S3

[7] J. Jiruše, M. Haničinec, M. Havelka, O. Hollricher, W. Ibach, P. Spizig Journal of Vacuum Science \& Technology B, 32 (2014), p. 06FC03-1

[8] M.D. Uchic, L. Holzer, B.J. Inkson, E.L. Principe and P. Munroe, MRS Bulletin 32(5) (2007), p. 408
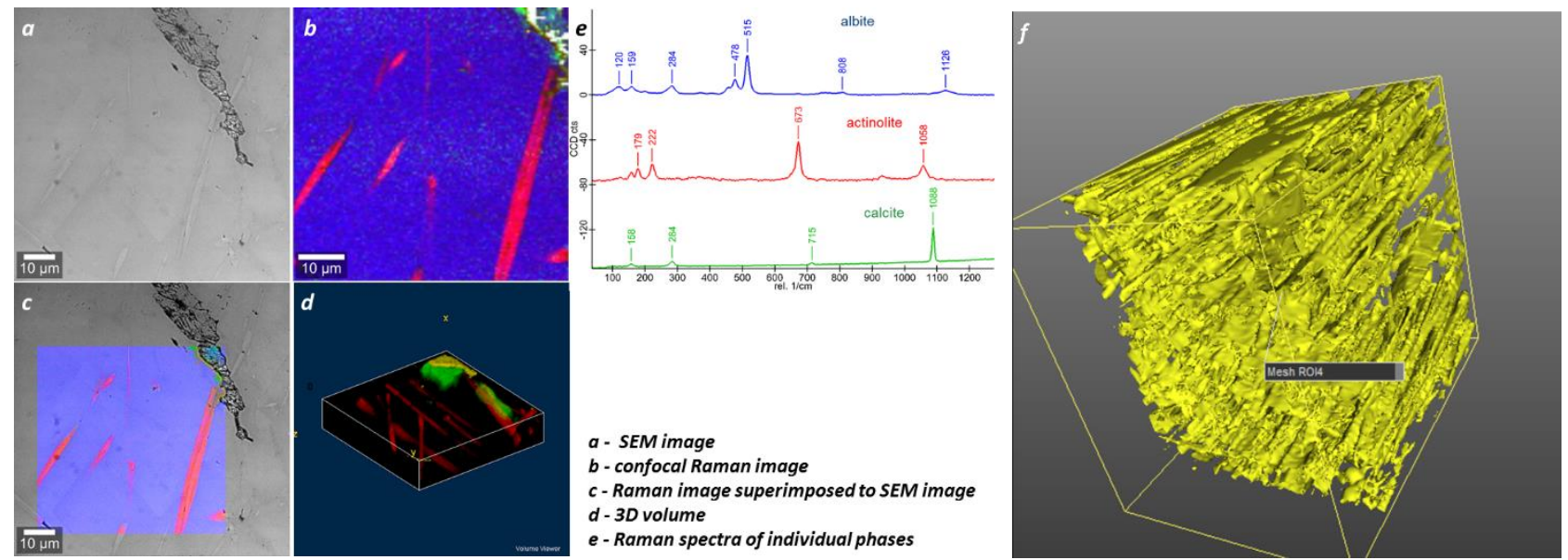

Figure 1. Actinolite fibers included in a mineral matrix. a- SEM image; b- Raman image; c- Raman image superimposed upon the SEM image d- 3D volume (Raman); e- Raman spectra of the individual phases (with corresponding color); f- 3D volume of a bundle in an equivalent sample (using SEM-FIB) 